\title{
Exosomes secreted by adipose-derived mesenchymal stem cells regulate type I collagen metabolism in fibroblasts from women with stress urinary incontinence
}

\author{
Xiaochun Liu ${ }^{1 *} \mathbb{B}$, Shiwei Wang${ }^{2}$, Suhui Wu', Qian Hao ${ }^{1}$, Ying $\mathrm{Li}^{1}$, Zhuodan Guo ${ }^{1}$ and Wenzhen Wang
}

\begin{abstract}
Background: Mesenchymal stem cells (MSC) have gained credibility as a therapeutic tool partly due to their potential to secrete factors such as cytokines and chemokines. Recently, exosomes, which mediate intercellular communication by delivering biomolecules such as mRNA and miRNA into recipient cells, have gained attention as a new and valuable therapeutic strategy in regenerative medicine. However, the potential role of exosomes secreted by adipose-derived mesenchymal stem cells (adMSC-Exos) in collagen metabolism is not well understood. The purpose of this study was to evaluate the effects of adMSC-Exos on collagen metabolism in cultured fibroblasts from women with stress urinary incontinence (SUI).
\end{abstract}

Methods: Periurethral vaginal wall tissues of postmenopausal women with or without SUI were collected during transvaginal surgical procedures. Primary fibroblasts were cultured from periurethral vaginal wall tissues, and the levels of type I collagen mRNA and protein were examined by qRT-PCR and western blotting. MSC were isolated from human adipose tissue by enzymatic digestion. Exosomes were prepared by ultracentrifugation of adMSCconditioned medium (adMSC-CM) and were confirmed by transmission electron microscopy and western blot analyses. The effects of adMSC-CM and adMSC-Exos were assessed using qRT-PCR and western blotting.

Results: The type I collagen content was significantly decreased in periurethral vaginal wall tissues and cultured vaginal fibroblasts from women with SUI. adMSC-CM increased the expression of the colla1 gene in vaginal fibroblasts from women with SUI. adMSC-Exos could be successfully isolated from adMSC-CM and could be transferred to fibroblasts efficiently. adMSC-Exos increased the expression of col1a1 in vaginal fibroblasts from women with SUI, and when the fibroblasts were treated with adMSC-Exos, the expression levels of TIMP-1 and TIMP-3 in fibroblasts were upregulated, with significant downregulation of MMP-1 and MMP-2 expression levels.

Conclusions: adMSC-Exos increased type I collagen contents by increasing collagen synthesis and decreasing collagen degradation in vaginal fibroblasts from women with SUI. adMSC-Exos may be a novel therapeutic approach for treating SUI.

Keywords: Mesenchymal stem cells, Exosomes, Col1a1, Collagen metabolism

\footnotetext{
* Correspondence:

${ }^{1}$ Department of O/G, Shanxi Academy of Medical Sciences \& Shanxi Da Yi

Hospital, Shanxi Da Yi Hospital Affiliated to Shanxi Medical University,

Taiyuan 030032, China

Full list of author information is available at the end of the article
}

(c) The Author(s). 2018 Open Access This article is distributed under the terms of the Creative Commons Attribution 4.0 International License (http://creativecommons.org/licenses/by/4.0/), which permits unrestricted use, distribution, and reproduction in any medium, provided you give appropriate credit to the original author(s) and the source, provide a link to the Creative Commons license, and indicate if changes were made. The Creative Commons Public Domain Dedication waiver (http://creativecommons.org/publicdomain/zero/1.0/) applies to the data made available in this article, unless otherwise stated. 


\section{Background}

Stress urinary incontinence (SUI), the most common form of incontinence, is defined as involuntary leakage of urine with effort or exertion, such as coughing or sneezing [1]. SUI is a major health problem affecting a large number of women of reproductive and menopausal ages and imposes a significant financial burden on health systems worldwide [2,3]. The underlying pathology and cause of SUI remain unknown, but many studies suggest that the development of SUI in women is accompanied by fibroblast dysfunction and reduced collagen [4-8].

Surgical treatment is the current gold standard therapy for SUI, and short-term success has been achieved with injectable bulking agents $[9,10]$. However, complications and poor long-term efficacy cause patients to seek alternative treatments, especially those that may provide safer and longer-lasting outcomes for SUI [11-13]. Recently, stem cell therapy has been extensively investigated at the experimental level and in a variety of clinical applications [14, 15]. Given its ability to induce tissue regeneration, stem cell-based treatment represents a promising strategy to overcome the limitations of current treatments for SUI.

Mesenchymal stem cells (MSC) are currently the most advanced and safe cell therapy tool for several diseases because of their easy isolation, high expansion potential resulting in an unlimited pool of transplantable cells, low immunogenicity, amenability to ex vivo genetic modification, and multipotency [16-19]. During the past 10 years, many pre-clinical and clinical studies on stem cells for SUI treatment have been published. For example, Zou et al. developed a tissue-engineered sling with bone marrow-derived mesenchymal stem cells seeded in a degradable silk scaffold and found that the tissue-engineered sling showed convincing functional effects for the treatment of stress urinary incontinence in a rat model [20]. Zhou et al. found that implantation of ADSCs via ureth$\mathrm{ral} /$ intravenous injection significantly decreased the abnormal voiding rate of an SUI rat model compared to the control group [21]. Lee et al. reported that intrinsic sphincter deficiency and mixed stress incontinency were effectively improved in 10 of 39 women with various types of stress urinary incontinence after transurethral umbilical cord blood stem cell injection [22]. All the results above indicate the regenerative potential of MSC for the treatment of SUI, but our understanding of how MSC mediate their effects on the continence mechanism is limited.

MSC are classically thought to exert a tissue repair effect through their capacity for multilineage differentiation and self-renewal. Indeed their regeneration and repair effects have been mainly attributed to their secreted factors rather than their multilineage differentiation capacity $[23,24]$. Exosomes which are defined as nanosized membrane vesicles with a diameter of $30-100 \mathrm{~nm}$ have been recently isolated from MSC-conditioned medium and these stem cell-derived exosomes are suggested to be the key intercellular communication mediators between MSC and target cells and to be associated with numerous physiological and pathological functions [25-28]. Here, to better understand the exosome-mediated intercellular communication between MSC and fibroblasts from women with SUI relative to collagen metabolism we first isolated and characterized human adipose-derived MSC (adMSC-Exos). Then we investigated the roles of exosomes secreted by adMSC-Exos in collagen metabolism. We observed that adMSC-Exos can promote the proliferation of fibroblasts. Furthermore advanced studies have shown that adMSC-Exos promoted collagen synthesis and inhibited collagen degradation by modulating the expression levels of TIMP metallopeptidase inhibitor 1 (TIMP-1) matrix metallopeptidase 1 (MMP-1) and type I collagen. To the best of our knowledge, this is the first report to show that adMSC-Exos can regulate collagen metabolism in fibroblasts from women with SUI. Because adMSC-Exos upregulate the concentration of type I collagen in fibroblasts from women with SUI they may represent a potential treatment candidate for SUI.

\section{Methods}

\section{Tissue collection}

Periurethral vaginal wall tissues were obtained from SUI $(+)(n=12)$ and SUI( $(-)(n=12)$ patients. A signed informed consent form was obtained from all participating women. Women with a history of endometriosis, gynecologic malignancies, connective tissue disorders, prior pelvic surgery, and advanced pelvic organ prolapse (higher than stage II on the Pelvic Organ Prolapse Quantification system) were excluded. Approximately $1 \mathrm{~cm}^{2}$ of full-thickness periurethral vaginal wall was excised $1 \mathrm{~cm}$ lateral to the urethrovesical junction from women undergoing surgery for SUI. Biopsies $\left(1 \mathrm{~cm}^{2}\right)$ of vaginal walls from a similar area were excised from controls undergoing transvaginal gynecologic surgery.

\section{Cell culture}

We isolated fibroblasts from vaginal wall tissues and cultured them as described previously [29]. The purity of cultured fibroblasts was confirmed by immunofluorescence using mouse anti-alpha smooth muscle actin antibody monoclonal antibody (1:1000; Abcam, Cambridge, MA, USA). Fibroblasts isolated from each patient's vaginal wall tissue were cultured in 6-well plates (BD Falcon, Franklin Lakes,NJ,USA) in triplicate. Cells from passage 3 were used in the experiments.

Adipose tissue was obtained from human liposuction aspirates with informed consent from the donors (healthy women and SUI patients). Isolation of adMSC was performed as previously reported [30]. Human 
adMSC were resuspended in the culture medium and seeded at a density of $2 \times 10^{6}$ cells per dish $(10 \mathrm{~cm})$. Cultures were maintained in a $37{ }^{\circ} \mathrm{C}$ incubator with $5 \%$ $\mathrm{CO} 2$ and passaged with trypsin/ethylenediaminetetraacetic acid when the cells were $70 \%$ confluent.

\section{Collagen synthesis assay}

The percent of collagen synthesized (as a percent of total protein) was determined [31]. The cells were washed and incubated for $3 \mathrm{~h}$ at $37{ }^{\circ} \mathrm{C}$ with $2,3-{ }^{3} \mathrm{H}$-proline in medium containing ascorbic acid $(50 \mathrm{mg} / \mathrm{mL})$. Cells and media were collected, and the protein was precipitated overnight with $20 \%$ trichloroacetic acid (TCA) at $4{ }^{\circ} \mathrm{C}$. The precipitate was collected by centrifugation at $3000 \mathrm{rpm}$ for $10 \mathrm{~min}$, and the obtained pellet was washed three times with $5 \% \mathrm{TCA} / 0.01 \%$ proline. The resulting pellet was dissolved in $0.2 \mathrm{M} \mathrm{NaOH}$, and the solution was titrated to a $\mathrm{pH}$ of 7.6 with $0.2 \mathrm{M} \mathrm{HCl}$. This solution was divided into two aliquots. One aliquot was digested with $100 \mathrm{~mL}$ of collagenase $(2 \mathrm{mg} / \mathrm{mL}$; Sigma-Aldrich, St Louis, MO, USA). The other aliquot was incubated in the buffer (without collagenase) for $1 \mathrm{~h}$ at $37{ }^{\circ} \mathrm{C}$. Proteins were precipitated with $10 \%$ TCA and $0.5 \%$ tannic acid for $1 \mathrm{~h}$ on ice. The precipitate was separated from the supernatant by centrifugation at 10,000 rpm for $5 \mathrm{~min}$. The supernatants were retained, and the pellets were dissolved in $0.2 \mathrm{M} \mathrm{NaOH}$. Radioactivity in the supernatants and the dissolved pellets was determined by liquid scintillation counting. Synthesized collagen was considered a collagenase releasable count (supernatant of the collagenase-treated aliquot). Total protein was the value of collagenase releasable counts plus collagenase insensitive counts (pellet of the collagenase-treated aliquot). A correction factor of 5.4 was applied to the collagenase insensitive counts to correct for the relative abundance of proline and hydroxyproline. Collagen synthesis is reported as a percent of the total protein synthesized.

\section{Western blot analysis}

Sodium dodecyl sulfate (SDS) sample buffer (0.5 M Tris- $\mathrm{HCl}, \mathrm{pH} 6.8,20 \%$ sucrose, $10 \%$ SDS, $1 \%$ bromophenol blue) was added to the concentrated samples. After equal amounts of protein ( $5 \mu \mathrm{g} / \mathrm{lane})$ were separated on 4-20\% gradient polyacrylamide gels (Bio-Rad, Hercules, CA, USA) under nonreducing conditions, the gels were blotted onto nitrocellulose membranes (Pierce, Waltham, MA, USA) in an electrophoretic transfer cell (Bio-Rad). Blots were blocked with 5\% non-fat milk at $4{ }^{\circ} \mathrm{C}$ overnight and then probed with Anti-Collagen I antibody (1:1000; Abcam, Cambridge, UK, ab23446), anti-CD63 antibody (1:1000; Abcam, ab59479), anti-Hsp70 antibody (1:1000; Abcam, ab2787) and anti-CD81 antibody (1:1000; Abcam, ab59477) at room temperature for $1 \mathrm{~h}$. After three washes with PBS-T $(\mathrm{pH} 7.4$ and $0.1 \%$ Triton), the membrane was incubated with sheep anti-mouse IgG conjugated to HRP (NeoBioscience, Shenzhen, China) for $1 \mathrm{~h}$ at room temperature, followed by three washes in PBS-T. The band density was determined by Bio-Rad Quality One Software.

\section{Real-time RT-PCR}

Total RNA was extracted with a Total RNA Isolation Kit (Takara Bio, Shiga, Japan) according to the manufacturer's instructions and quantified using spectrophotometry (NANO drop 2000; Thermo Fisher Scientific, Waltham, MA, USA). The primer sequences were: Colla1, 5'-GAGG GCCAA GACGAAGACATC-3' (sense) and 5'-CAGATCA CGTCATCGCACAAC-3' (anti-sense); MMP-1, 5'-AAAA TTACACGCCAGATTTGCC-3' (sense) and 5'-GGT GT GACATTACTCCAGA GTTG-3' (anti-sense); MMP-2, 5'-GATACCCCTTTGA CGGTAAGGA-3' (sense) and 5'-CCTTCTCCCAAGGTCCATAGC-3' (anti-sense); MM P-9, 5'-AGACCTGGGCAGATTCCAAAC-3' (sense) and 5'-CGGCAAGTCTT CCGAGTAGT-3' (anti-sense); TIM P-1, 5'-AGAGTGTCTGCGGATACTTCC-3' (sense) and 5'-CCAACAGTGTAGGTCTTGGTG-3' (anti-sense); TIM P-2, 5'-GCTG CGAGTGCAAGATCAC-3' (sense) and 5'-TGGTGCCCGTTGATGTTCTTC-3' (anti-sense); TIM P-3, 5'-CATGTGCAGTACATCCATACGG-3' (sense) and 5'-CATC ATAGACGCGACCTGTCA-3' (anti-sense). The relative expression of mRNAs was evaluated by the $2^{-\Delta \Delta C t}$ method and normalized to the expression of GAPDH. The cycling program involved preliminary denaturation at $95^{\circ} \mathrm{C}$ for $1 \mathrm{~min}$, followed by 45 cycles of denaturation at $95^{\circ} \mathrm{Cfor}$ $10 \mathrm{~s}$, annealing at $62{ }^{\circ} \mathrm{Cfor} 25 \mathrm{~s}$, and elongation at $62{ }^{\circ} \mathrm{C}$ for $20 \mathrm{~s}$, followed by a final elongation step at $70{ }^{\circ} \mathrm{C}$ for $5 \mathrm{~min}$.

\section{Immunofluorescence staining}

Fibroblasts were fixed at room temperature with $4 \%$ PFA for $10 \mathrm{~min}$. After permeabilization in 1\% Triton X-100/ PBS for $15 \mathrm{~min}$, nonspecific binding was blocked with $3 \%$ bovine serum albumin for $1 \mathrm{~h}$ at $37{ }^{\circ} \mathrm{C}$. Then, fibroblasts were incubated in mouse anti-alpha smooth muscle actin antibody monoclonal antibody (1:1000; Abcam, ab119952) at the appropriate dilution at $4{ }^{\circ} \mathrm{C}$ overnight. Secondary antibodies were used for $1 \mathrm{~h}$ at $37^{\circ} \mathrm{C}$ after washing with PBS. Hoechst counterstain was used for visualization. Pictures were captured using an Olympus inverted fluorescence microscope (Olympus, Tokyo, Japan).

\section{Flow cytometric analysis of cell immunophenotype}

For immunophenotype analysis of adMSC, cells were trypsinized and washed with $2 \mathrm{~mL}$ of phosphate-buffered saline (PBS) containing $0.5 \%$ bovine serum albumin (BSA; Sigma) per tube. Cells were counted and centrifuged at $1200 \mathrm{~g}$ for 
$5 \mathrm{~min}$. After discarding the supernatant, the cell pellet was resuspended in $50 \mu \mathrm{L}$ of PBS containing fluorescein isothiocyanate (FITC)-labeled primary antibodies and incubated at $4{ }^{\circ} \mathrm{C}$ for $30 \mathrm{~min}$. FITC-labeled immunoglobulin G1 (IgG1) of the same species and isotypes was used as negative control. Finally, the cells were washed twice with $2 \mathrm{~mL}$ of PBS containing 0.5\% BSA and resuspended in $0.5 \mathrm{~mL}$ of $\mathrm{PBS}$ for fluorescence-activated cell sorting (FACS) analysis. The working concentrations of the primary antibodies against human CD29 (eBioscience, San Diego, CA, USA; 11-0299-42), CD31 (eBioscience; 11-0319-42), CD34 (eBioscience; 110349-42), CD44 (eBioscience; MA1-10228), CD105 (eBioscience; MA1-19594) and HLA-DR (eBioscience; 11-9956-42) were 10-20 ng/mL. Flow cytometric analysis was performed with a flow cytometer BD Accuri ${ }^{\mathrm{im}} \mathrm{C6}$ (BD Biosciences, Franklin Lakes, NJ, United States). Data were analyzed with CFlow Plus 1.0 (BD Biosciences).

\section{Multilineage differentiation of adMSC}

To identify the capacity for adMSC multilineage differentiation, adMSCs were cultured under differentiation conditions. For adipocyte differentiation, adMSCs were cultured at $2-310^{4} / \mathrm{cm}^{2}$ in DMEM with $10 \% \mathrm{FCS}, 10^{-6} \mathrm{M}$ dexamethasone, $50 \mathrm{mg} / \mathrm{mL}$ ascorbic acid, and $100 \mathrm{mg} / \mathrm{mL}$ 1-methyl-3-isobutyl-xanthine (all from Sigma-Aldrich). After 14 days, Oil Red O staining was performed to show adipocyte differentiation. For osteoblast differentiation, adMSCs were cultured at $10^{4} / \mathrm{cm}^{2}$ in DMEM with $10 \%$ FBS, $10 \mathrm{mM} \beta$-glycerophosphate, $10^{-7} \mathrm{M}$ dexamethasone, and $0.2 \mathrm{mM}$ ascorbic acid (all from Sigma-Aldrich). After 21 days, the cells were stained with ALP staining to reveal osteogenic differentiation.

\section{Exosome extraction}

Exosome extraction was performed as previously described [32]. AdMSC-Exos were generated from adMSCs of healthy women, and SUI-Exos were generated from adMSCs of SUI patients. Briefly, adMSCs were cultured for $48 \mathrm{~h}$ before exosome isolation. Then, the medium was harvested and centrifuged at $800 \mathrm{~g}$ for $5 \mathrm{~min}$, followed by $2000 \mathrm{~g}$ for $10 \mathrm{~min}$ to remove the lifted cells. The supernatants were subjected to filtration on a $0.1-\mathrm{mm}$-pore polyethersulfone membrane filter (Corning, Corning, NY, USA) to discard large vesicles and cell debris. Then, the mixtures were concentrated by a $100,000-\mathrm{Mw}$ cutoff membrane (Centricon Plus-70, Merck Millipore, Burlington, MA, USA). The volume of the supernatants was reduced from approximately $250-500 \mathrm{~mL}$ to $30 \mathrm{~mL}$. Then, the supernatants were ultracentrifuged at 100,000 g for $1 \mathrm{~h}$ at $4{ }^{\circ} \mathrm{C}$ using a $70 \mathrm{Ti}$ rotor (Beckman Coulter, Brea, CA, USA). Exosomes were then resuspended in $6 \mathrm{~mL}$ of PBS and ultracentrifuged at $100,000 \mathrm{~g}$ for $1 \mathrm{~h}$ at $4{ }^{\circ} \mathrm{C}$ using a 100Ti rotor (Beckman Coulter).

\section{Dil-labeled exosomes to fibroblasts}

Purified adMSC-Exos were labeled with $1 \mu \mathrm{M}$ of Dil (Invitrogen, Waltham, MA, USA) as previously described [33]. Pelleted exosomes were washed to remove unbound Dil, resuspended in $\mathrm{PBS} / 5 \% \mathrm{BSA}$, and then added to fibroblast medium for $6 \mathrm{~h}$. Fibroblasts were then washed in PBS, fixed in $4 \%$ paraformaldehyde, and imaged by microscopy.

\section{Transmission electron microscopy}

Purified exosomes were fixed with $1 \%$ glutaraldehyde in PBS (pH 7.4). After rinsing, a $20-\mu \mathrm{L}$ drop of the suspension was loaded onto a formvar/carbon-coated grid negatively stained with $3 \%(w / v)$ aqueous phosphotungstic acid for $1 \mathrm{~min}$ for observation by transmission electron microscopy (Hitachi, Tokyo, Japan, SU-8010).

\section{Statistical analysis}

All values are shown as the mean \pm s.d. Differences between two groups were determined with the unpaired Student's $t$ test. ANOVA was used for multiple comparisons. $P<0.05$ was considered significant.

\section{Results}

Collagen concentration in periurethral vaginal wall tissues and cultured vaginal fibroblasts from women with or without SUI

To investigate collagen expression levels in periurethral vaginal wall tissues of postmenopausal women with or without SUI, vaginal wall tissues were collected from women with SUI during transvaginal surgical procedures (Table 1). The total collagen concentration in periurethral vaginal wall tissues from women with SUI was significantly downregulated compared to that in the tissues from women without SUI $(P<0.05$; Fig. 1a). qRT-PCR analysis showed that the expression levels of Col1a1 were significantly reduced in the women with

Table 1 Case description

\begin{tabular}{llll}
\hline & $\begin{array}{l}\text { With SUI } \\
(n=12)\end{array}$ & $\begin{array}{l}\text { Without SUI } \\
(n=12)\end{array}$ & $P$ value \\
\hline Age, yrs & $63.08 \pm 5.93$ & $62.50 \pm 8.15$ & 0.843 \\
Parity, n & $2.67 \pm 0.89$ & $2.50 \pm 0.80$ & 0.633 \\
Mode of labor & Vaginal & Vaginal & \\
Birth weight, g & $3345.83 \pm 542.49$ & $3283.22 \pm 518.45$ & 0.776 \\
BMl, kg/m2 & $31.07 \pm 2.83$ & $29.33 \pm 3.60$ & 0.201 \\
Menopausal duration, yrs & $8.42 \pm 2.19$ & $8.50 \pm 2.39$ & 0.930 \\
\hline
\end{tabular}

The data of age, parity, birth weight, body mass index (BMI) and menopause duration of 12 patients in each group were compared, and no differences were detected 

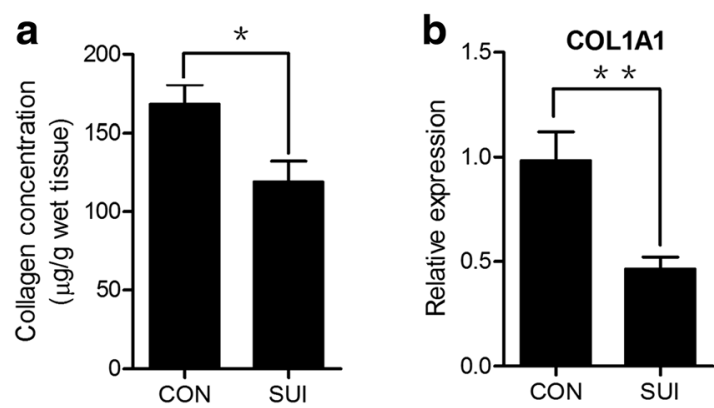

C

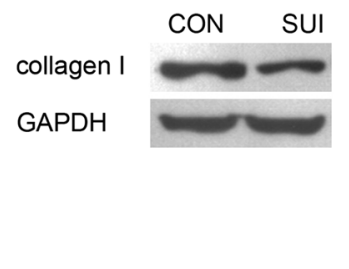

d

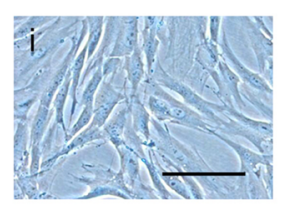

e
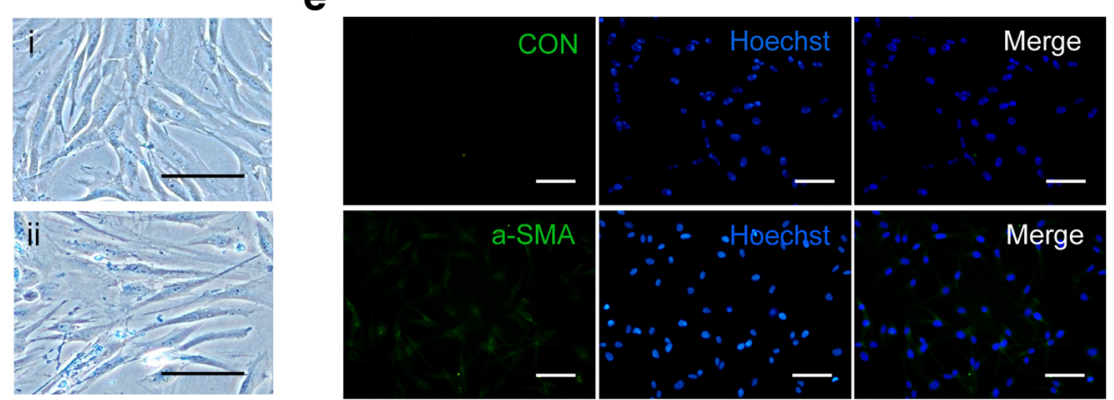

f
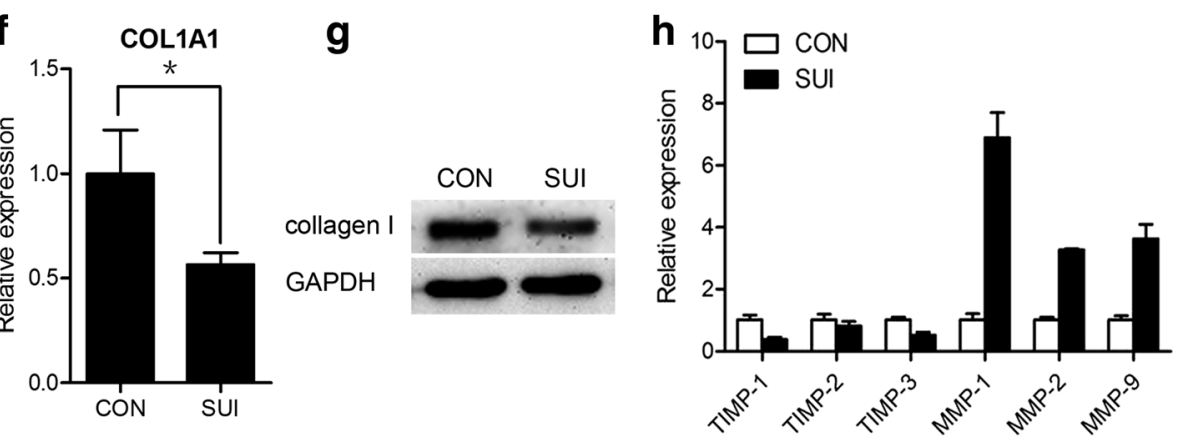

Fig. 1 Collagen concentration in periurethral vaginal wall tissues and cultured vaginal fibroblasts from women with or without SUI. a Total collagen concentration measured as micrograms of hydroxyproline per milligrams of wet weight from urogenital tissues of women with SUI and in urologically healthy women. The results are the mean \pm s.d. ( $n=12$ for each group). ${ }^{*} P<0.05$. $\mathbf{b}$ The levels of Col 1 a 1 in urogenital tissues of women with SUI and in urologically healthy women were analysed by qRT-PCR. ${ }^{*} P<0.01$. $\mathbf{c}$ The expression of type I collagen in urogenital tissues was determined by a western blot assay. $\mathbf{d}$ The morphology of cultured vaginal fibroblasts in women with SUI (ii) and in urologically healthy women (i). e a-SMA protein expression by immunofluorescence staining (green). Original scale bars $=50 \mu \mathrm{m}$. $\mathbf{f}$ The levels of Col1a1 in cultured vaginal fibroblasts from women with or without SUI were analyzed by qRT-PCR. The results are the mean \pm s.d. ( $n=3$ for each group). ${ }^{*} P<0.05$. $\mathbf{g}$ The expression of type I collagen in cultured vaginal fibroblasts was determined by a western blot assay. $\mathbf{h}$ The levels of TIMP-1, TIMP-2, TIMP-3, MMP-1, MMP-2, and MMP-9 in fibroblasts from women with or without SUl were analyzed by qRT-PCR. The results are the mean \pm s.d. ( $n=3$ for each group). CON control, MMP-1 matrix metallopeptidase 1, MMP-3 matrix metallopeptidase 3, MMP-9 matrix metallopeptidase 9, SUI stress urinary incontinence, TIMP-1 TIMP metallopeptidase inhibitor 1, TIMP-2 TIMP metallopeptidase inhibitor 2, TIMP-3 TIMP metallopeptidase inhibitor 3

SUI $(P<0.01$; Fig. 1b). Western blotting also demonstrated that type I collagen was significantly reduced in the patients with SUI (Fig. 1c).

Collagen is mainly synthesized by fibroblasts in periurethral vaginal wall tissue. To examine collagen metabolism changes in fibroblasts from women with SUI, fibroblasts were isolated from periurethral vaginal wall tissues of women with SUI and from healthy women. We found that cultured vaginal fibroblasts from the women with SUI showed more elongation than those from the healthy women (Fig. 1d). Immunostaining assay identified the fibroblast marker a-smooth muscle actin (a-SMA) in these fibroblasts as expected (Fig. 1e). qRT-PCR analysis showed that the expression of Colla1 was significantly reduced in the patients with SUI (Fig. 1f). Western blotting also demonstrated that type I collagen was significantly reduced in the patients with SUI (Fig. 1g). Furthermore, we found that fibroblasts from the women with SUI exhibited significant decreases in the expression levels of TIMP-1, TIMP-2 and 
TIMP-3, while the mRNA expression levels of MMP-1, MMP-2, and MMP-9, which reportedly increase collagen degradation in stress incontinence, were significantly increased (Fig. 1h). Taken together, these data suggested that the type I collagen content was significantly decreased in periurethral vaginal wall tissues and cultured vaginal fibroblasts from the women with SUI.

\section{Characterization of human adMSC}

MSC were initially isolated from the bone marrow; however, the isolation of bone marrow MSC requires invasive aspiration from a donor, significantly restricting its application. Because adipose tissue is another rich source of MSC (adMSC) and enables auto-grafting, adipose tissue has recently become a popular source for MSC isolation. In this study, to study the effects of MSC on the collagen metabolism of fibroblasts, we isolated MSC from human adipose tissues. We found that human adMSC have a characteristic morphology of fibroblast-like cells (Fig. 2b).
They express high levels of CD29, CD44, and CD105, but they are persistently negative for CD31, CD34, and HLA-DR (Fig. 2a) as previously reported [30]. Under appropriate culture conditions, adMSC can differentiate into adipocytes and osteoblasts as demonstrated by Oil red $\mathrm{O}$ staining and ALP staining, respectively (Fig. 2c, d).

\section{adMSC-conditioned medium regulates type I collagen metabolism in cultured vaginal fibroblasts from women with SUI}

To study the effect of adMSC on collagen metabolism, fibroblasts from women with SUI were treated with adMSC-conditioned medium for $48 \mathrm{~h}$. qRT-PCR and western blotting analysis showed that the expression of Colla1 in fibroblasts from the women with SUI was significantly increased after treatment with adMSC-conditioned medium (Fig. 3a, b). Furthermore, when the fibroblasts were treated with adMSC-conditioned medium, we found that the expression levels of TIMP-1 and TIMP-3 were significantly
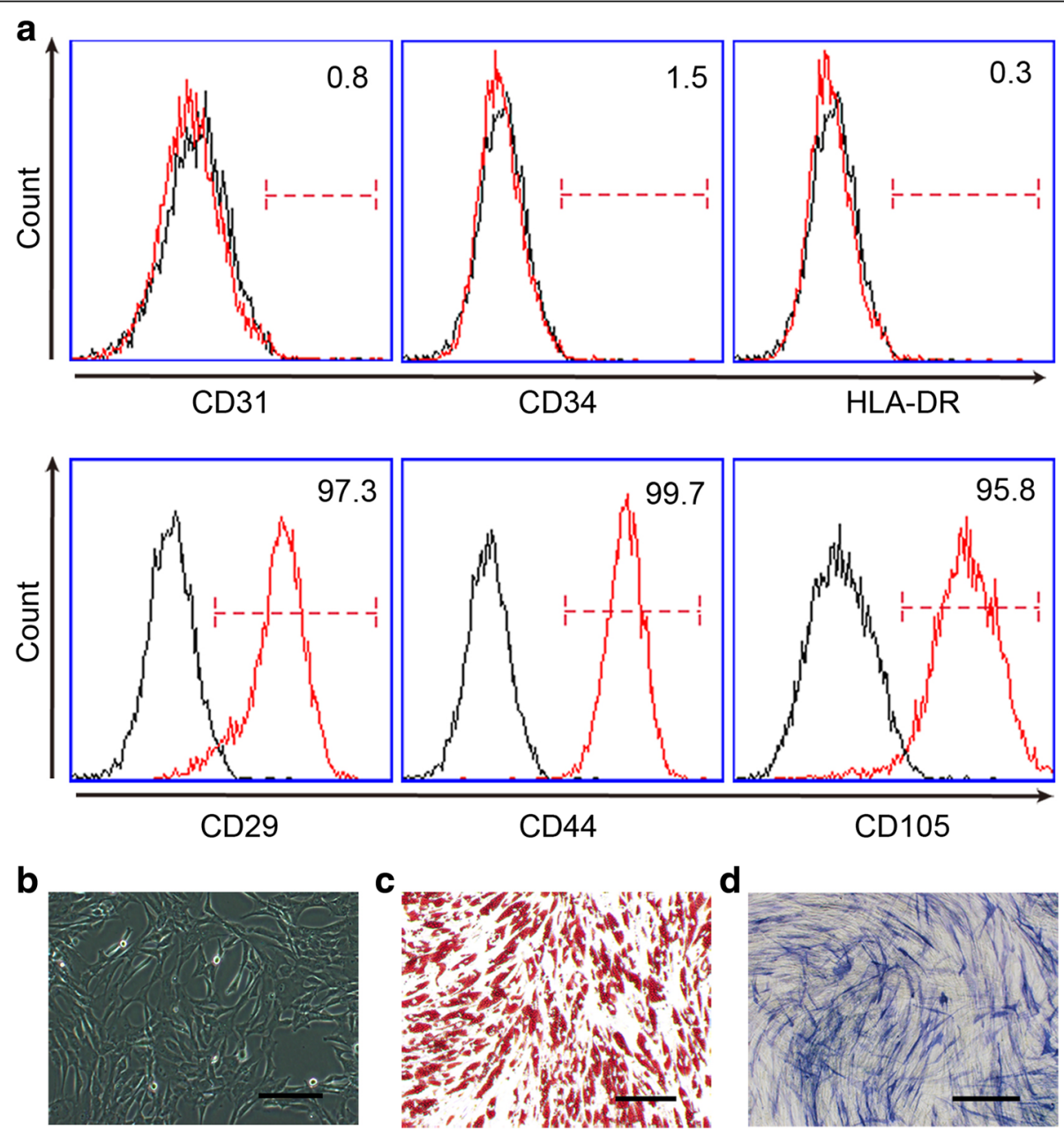

Fig. 2 Characterization of human adMSC. a Immunophenotype of human adMSC. b The morphology of human adMSC was observed under light microscopy. Original scale bars $=50 \mu \mathrm{m}$. c The differentiation capacity of human adMSC was demonstrated by Oil red O staining for adipocytes (c) and ALP staining for osteoblasts (d). Original scale bars $=50 \mu \mathrm{m}$ 

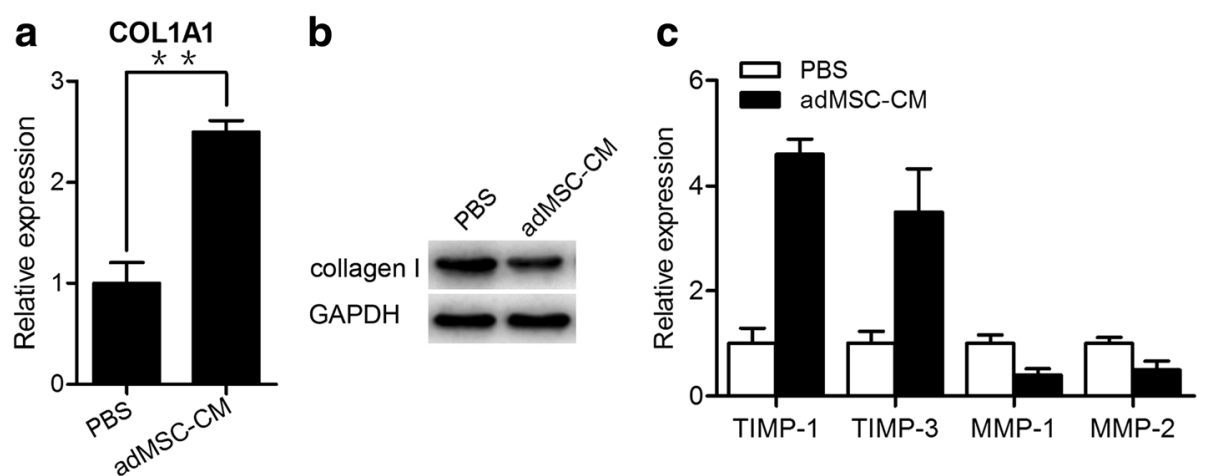

Fig. 3 adMSC-conditioned medium regulates type I collagen metabolism in cultured vaginal fibroblasts from women with SUI. a Fibroblasts were incubated with adMSC-conditioned medium for $48 \mathrm{~h}$. The mRNA levels of col1a1 were evaluated by qRT-PCR. The results are the mean \pm s.d. ( $n=3$ for each group). ${ }^{*} P<0.01$. b The expression of type I collagen was determined by a western blot assay. c The mRNA levels of TIMP-1, TIMP-3, MMP-1, and MMP-2 were evaluated by qRT-PCR. The results are the mean \pm s.d. ( $n=3$ for each group). adMSC-CM adipose-derived mesenchymal stem cell-conditioned medium, MMP-1 matrix metallopeptidase 1, MMP-3 matrix metallopeptidase 3, TIMP-1 TIMP metallopeptidase inhibitor 1, TIMP-2 TIMP metallopeptidase inhibitor 2

increased, while the mRNA expression levels of MMP-1 and MMP-2, which reportedly decrease collagen degradation in stress incontinence, were significantly decreased (Fig. 3c). Therefore, our results indicated that adMSC-conditioned medium regulated collagen metabolism by increasing collagen synthesis and decreasing collagen degradation in vaginal fibroblasts in women with SUI.

\section{Isolation and characterization of adMSC-Exos}

To study the roles of exosomes in collagen metabolism, adMSC-Exos were first isolated and characterized as previously described [33]. Transmission electron microscopy analysis showed that the exosomes purified from the adMSC-conditioned medium were round membrane-bound vesicles with a size ranging from 30 to $100 \mathrm{~nm}$ in diameter (Fig. 4a). Western blot demonstrated that the exosome marker proteins CD63, HSP70, and CD81 were present in adMSC-Exos as expected (Fig. 4b). To further investigate whether the adMSC-Exos could be transferred to fibroblasts, adMSC-Exos were labeled with Dil dye and incubated with fibroblasts from women with SUI in vitro. The uptake was confirmed by fluorescence microscopy. After $8 \mathrm{~h}$, more than $90 \%$ of the fibroblasts were Dil-positive; the Dil-labeled adMSC-Exos had been taken up and were transferred to cytoplasm compartments (Fig. 4c). These data suggested that adMSC-Exos were successfully isolated and could be efficiently transferred to fibroblasts.

\section{adMSC-Exos regulate type I collagen metabolism in cultured vaginal fibroblasts from women with SUI}

To examine the effects of adMSC-Exos on collagen metabolism, fibroblasts from women with SUI were treated with exosomes for $48 \mathrm{~h}$. Our results showed that the expression levels of colla1 were increased in the fibroblasts incubated with adMSC-Exos compared to those of the fibroblasts incubated with SUI-Exos (Fig. 5a, b). Moreover, when the fibroblasts were treated with adMSC-Exos, the expression levels of TIMP-1 and TIMP-3 in the fibroblasts were upregulated, with significant downregulation of MMP-1 and MMP-2 (Fig. 5c). Taken together, our observations suggested that adMSC-Exos increased the type I collagen content by increasing collagen synthesis and decreasing collagen degradation in vaginal fibroblasts from women with SUI.

\section{Discussion}

Stress urinary incontinence (SUI), which is defined as involuntary leakage of urine during an increase in abdominal pressure, is a prevalent urological problem that is most common in women, with an incidence of $18.9 \%$ in those aged $>20$ years in China [34]. The cause of stress urinary incontinence is multifactorial. Previous studies have reported a decreased collagen content and altered morphologic features in the pelvic support tissues of women with stress urinary incontinence $[7,8]$. In the present study, we found that the expression of type I collagen in periurethral vaginal wall tissues of postmenopausal women with SUI was significantly decreased compared to that in tissues from women without SUI, which is consistent with the observations of Chen et al., who demonstrated a $60 \%$ lower collagen content in the vaginal wall tissues of women with SUI compared to that in tissues from age-matched continent women [35].

Mesenchymal stem cells (MSC), which have the capacity for self-renewal and differentiation into chondrocytes, osteoblasts, and adipocytes, can be isolated and easily expanded from tissues such as bone marrow, 
a

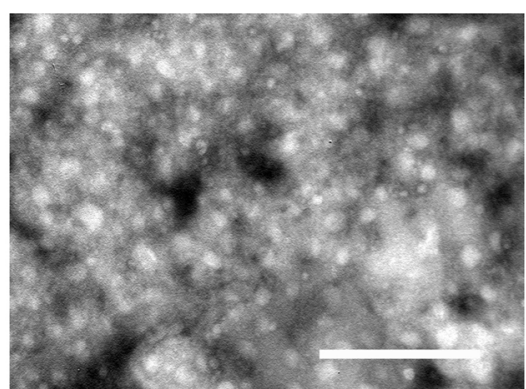

C
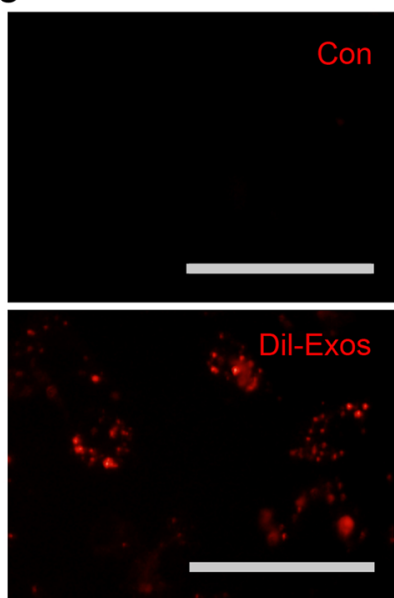
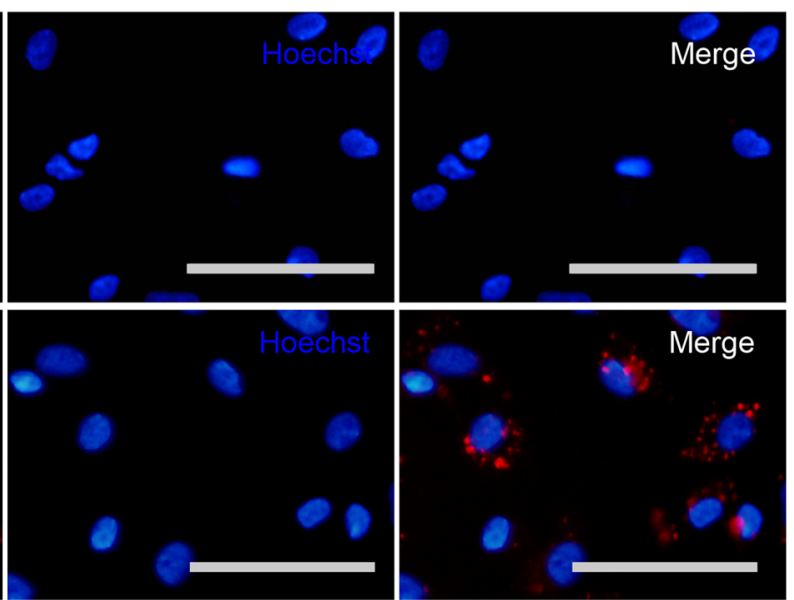

Fig. 4 Isolation and characterization of adMSC-Exos. a Electron micrograph of exosomes isolated from adMSC-conditioned medium. The arrowheads indicate exosomes. Original scale bars $=0.5 \mu \mathrm{m}$. b Western blotting was performed with adMSC-Exos (Exos) or adMSC-conditioned medium (Medium). CD63, HSP70 and CD81 expression levels in exosomes were detected. c Fibroblasts were incubated with Dil-labeled exosomes (Dil-Exos; Dil is shown in red) or carrier control (CON), and nuclei were stained with Hoechst 33,342 (blue). Original scale bars $=50 \mu \mathrm{m}$

adipose tissue, the umbilical cord, placenta, dental pulp and others [36, 37]. Here, we isolated MSC from human adipose tissues, adMSC, expressing cell surface markers such as CD29, CD44, and CD105, without the expression of other markers, including CD31, CD34, and HLA-DR surface molecules, and showing differentiation potential into osteocytes and adipocytes under in vitro conditions. It has been increasingly observed in various fields of regenerative medicine that transplanted MSC do not necessarily engraft and differentiate at the site of injury [38].
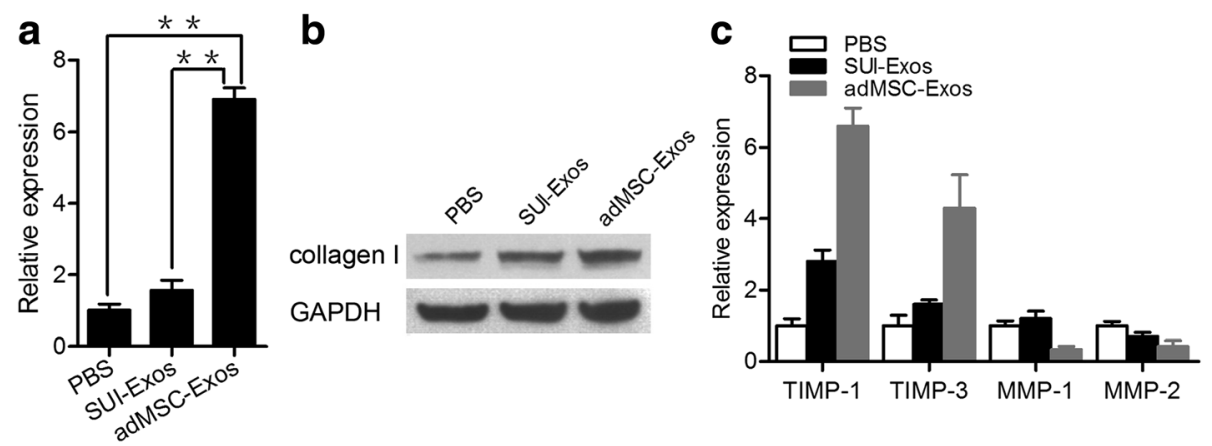

Fig. 5 adMSC-Exos regulate type I collagen metabolism in cultured vaginal fibroblasts from women with SUI. Fibroblasts were incubated with adMSC-Exos or SUI-Exos for $48 \mathrm{~h}$. a The levels of Col1a1 in cultured vaginal fibroblasts from women with SUI were analyzed by qRT-PCR. The results are the mean \pm s.d. ( $n=3$ for each group). ${ }^{*} P<0.01$. $\mathbf{b}$ The expression of type I collagen was determined by a western blot assay. $\mathbf{c}$ The levels of TIMP-1, TIMP-3, MMP-1, and MMP-2 in fibroblasts from women with SUI were analyzed by qRT-PCR. The results are the mean \pm s.d. ( $n=3$ for each group). adMSC-Exos exosomes secreted by adipose-derived mesenchymal stem cells, MMP-1 matrix metallopeptidase $1, M M P-3$ matrix metallopeptidase 3, SUI stress urinary incontinence, TIMP-1 TIMP metallopeptidase inhibitor 1, TIMP-2 TIMP metallopeptidase inhibitor 2 
Recent studies have shown that the key mechanism by which MSC contribute to tissue repair and regeneration is through their paracrine function [39, 40]. The present study found that the proliferation and type I collagen contents of fibroblasts from women with SUI increased after the fibroblasts were treated with adMSC-conditioned medium. Furthermore, we also found that under the influence of adMSC-conditioned medium, the expression levels of TIMP-1 and TIMP-3 were upregulated and the expression levels of MMP-1 and MMP-2 were downregulated. These results demonstrate that adMSC-conditioned medium regulated collagen metabolism in fibroblasts from women with SUI by increasing collagen synthesis and decreasing collagenolysis.

Recent studies have revealed that transplanted MSC may secrete abundant particles documented as exosomes, which are suggested to be central mediators of intercellular communication [41]. Since the first report of MSC-derived exosomes, a growing number of studies have explored their regenerative potential using different in vitro and in vivo models, and $\mathrm{Hu}$ et al. found that ASC-Exos could be taken up and internalized by fibroblasts to stimulate cell migration, proliferation and collagen synthesis [42]. In the present study, we successfully isolated and purified exosomes from human adMSC. In addition to demonstrating that Dil-labeled exosomes could be transferred to the cytoplasm of cultured fibroblasts from women with SUI, we further showed that the levels of type I collagen in cultured fibroblasts were significantly increased after the fibroblasts were treated with adMSC-Exos. We also found that adMSC-Exos upregulated the expression levels of TIMP-1 and TIMP-3 and downregulated the expression levels of MMP-1 and MMP-2. These results were consistent with those from adMSC-conditional medium, suggesting that adMSC-Exos play a crucial role in regulating collagen metabolism in fibroblasts from women with SUI.

\section{Conclusions}

To our knowledge, this is the first study demonstrating the modulation of collagen metabolism in fibroblasts from women with SUI by adMSC-Exos. Because exosomes are easier to preserve and transfer and have low immunogenicity, they are safe for therapeutic administration. Therefore, the use of adMSC-Exos provides an efficient and safe alternative to mesenchymal stem cell-based therapies in the treatment of SUI in the future.

\footnotetext{
Abbreviations

adMSC-CM: Adipose-derived mesenchymal stem cell-conditioned medium; adMSC-Exos: Exosomes secreted by adipose-derived mesenchymal stem cells; ALP: Alkaline phosphatase; MMP-1: Matrix metallopeptidase 1; MMP-2: Matrix metallopeptidase 2; MMP-9: Matrix metallopeptidase 9; MSC: Mesenchymal stem cells; SUI : Stress urinary incontinence; TIMP-1: TIMP metallopeptidase inhibitor 1; TIMP-2: TIMP metallopeptidase inhibitor 2; TIMP-3: TIMP metallopeptidase inhibitor 3
}

Availability of the data and materials

All data generated or analyzed during this study are included in this published article.

\section{Funding}

This work was supported by grants from the National Natural Science Foundation of China (No. 81401188) and Shanxi Provincial Natural Science Foundation of China (No. 2013011053-3).

\section{Authors' contributions \\ $X C L$ and SWW were responsible for the concept and design of the study. XCL was responsible for funding. XCL and SWW were responsible for participation in the study. SHW was responsible for arrangement of the patients and tissue sample collection. ZDG and WZW were responsible for cell culture and studies. ZDG and WZW were responsible for the preparation and characterization of exosomes. $\mathrm{QH}$ and $\mathrm{YL}$ were responsible for TEM. WZW and $\mathrm{QH}$ were responsible for the statistical analyses. XCL, SWW, QH, and ZDG were responsible for writing and critical reading of the manuscript. All authors read and approved the final manuscript.}

Ethics approval and consent to participate

This study was approved by the Research Ethics Committee of Shanxi Dayi Hospital, Taiyuan, Shanxi, China.

Consent for publication

All authors of this manuscript agreed to publication.

Competing interests

The authors declare that they have no competing interests.

\section{Publisher's Note}

Springer Nature remains neutral with regard to jurisdictional claims in published maps and institutional affiliations.

\section{Author details}

${ }^{1}$ Department of O/G, Shanxi Academy of Medical Sciences \& Shanxi Da Yi Hospital, Shanxi Da Yi Hospital Affiliated to Shanxi Medical University, Taiyuan 030032, China. ${ }^{2}$ School of Medicine, University of Tasmania, Hobart 7000, Australia.

Received: 6 March 2018 Revised: 12 April 2018

Accepted: 7 May 2018 Published online: 13 June 2018

\section{References}

1. Weber AM, Abrams P, Brubaker L, Cundiff G, Davis G, Dmochowski RR, et al. The standardization of terminology for researchers in female pelvic floor disorders. Int Urogynecol J Pelvic Floor Dysfunct. 2001;12:178-86.

2. Hunskaar S, Arnold EP, Burgio K, Diokno AC, Herzog AR, Mallett VT. Epidemiology and natural history of urinary incontinence. Int Urogynecol J Pelvic Floor Dysfunct. 2000;11:301-19.

3. Fusgen I. Urinary incontinence in old age-state of the art. Z Gerontol Geriatr. 2005;38(1):14-9.

4. Keane DP, Sims TJ, Abrams P, Bailey AJ. Analysis of collagen status in premenopausal nulliparous women with genuine stress incontinence. $\mathrm{Br}$ J Obstet Gynaecol. 1997;104:994-8.

5. Wong MY, Harmanli OH, Agar M, Dandolu V, Terry Grody MH. Collagen content of nonsupport tissue in pelvic organ prolapse and stress urinary incontinence. Am J Obstet Gynecol. 2003;189:1597-600.

6. FitzGerald MP, Mollenhauer J, Hale DS, Benson JT, Brubaker L. Urethral collagen morphologic characteristics among women with genuine stress incontinence. Am J Obstet Gynecol. 2000;182:1565-74.

7. Falconer C, Ekman G, Malmstrom A, Ulmsten U. Decreased collagen synthesis in stress incontinent women. Obstet Gynecol. 1994;84:583-6.

8. Rechberger T, Postawski K, Jakowicki JA, Gunja-Smith Z, Woessner JF. Role of fascial collagen in stress urinary incontinence. Am J Obstet Gynecol. 1998;179:1511-4.

9. Albo ME, Richter HE, Brubaker L, Norton P, Kraus SR, Zimmern PE, et al. Burch colposuspension versus fascial sling to reduce urinary stress incontinence. New Engl J Med. 2007;356(21):2143-5. 
10. Deng DY, Rutman M, Raz S, Rodriguez LV. Presentation and management of major complications of midurethral slings: Are complications underreported? Neurourol Urodyn. 2007;26:46-52.

11. Olsen AL, Smith VJ, Bergstrom JO, Colling JC, Clark AL. Epidemiology of surgically managed pelvic organ prolapse and urinary incontinence. Obstet Gynecol. 1997;89:501-6.

12. Rogers RG. What's best in the treatment of stress urinary incontinece? New Engl J Med. 2010;362(22):2124-5.

13. Gungorduk K, Celebi I, Ark C, Celikkol O, Yildirim G. Which type of midurethral sling procedure should be chosen for treatment of stress urinary incontinance with intrinsic sphincter deficiency? tension-free vaginal tape or transobturator tape. Acta Obstet Gynecol Scand. 2009;88(8):920-6.

14. Kim J, Lee S, Song Y, Lee H. Stem cell therapy in bladder dysfunction: where are we? And where do we have to go? Biomed Res Int. 2013;2013:930713.

15. Vaegler M, Lenis A, Daum L, Amend B, Stenzl A, Toomey P, et al. Stem cell therapy for voiding and erectile dysfunction. Nat Rev Urol. 2012;9:435-47.

16. Xu Y, Malladi P, Wagner DR, Longaker MT. Adipose-derived mesenchymal cells as a potential cell source for skeletal regeneration. Curr Opin Mol Ther. 2005;7(4):300-5.

17. Nagaya N, Kangawa K, Itoh T, Iwase T, Murakami S, Miyahara Y, et al. Transplantation of mesenchymal stem cells improves cardiac function in a rat model of dilated cardiomyopathy. Circulation. 2005;112(8):1128-35.

18. Fraser JK, Wulur I, Alfonso Z, Hedrick MH. Fat tissue: an underappreciated source of stem cells for biotechnology. Trends Biotechnol. 2006;24(4):150-4.

19. Jiang $Y$, Jahagirdar BN, Reinhardt RL, Schwartz RE, Keene CD, Ortiz-Gonzalez $X R$, et al. Pluripotency of mesenchymal stem cells derived from adult marrow. Nature. 2002;418(6893):41-9.

20. Zou XH, Zhi YL, Chen X, Jin HM, Wang LL, Jiang YZ, et al. Mesenchymal stem cells seeded knitted silk sling for the treatment of stress urinary incontinence. Biomaterials. 2010;31:4872-9.

21. Zhou S, Zhang K, Atala A, Khoury O, Murphy SV, Zhao W, et al. Stem cell therapy for treatment of stress urinary incontinence: the current status and challenges. Stem Cells Int. 2016;2016:7060975.

22. Lee CN, Jang JB, Kim JY, Koh C, Baek JY, Lee KJ. Human cord blood stem cell therapy for treatment of stress urinary incontinence. J Korean Med Sci. 2010;25:813-6.

23. Molloy AP, Martin FT, Dwyer RM, Griffin TP, Murphy M, Barry FP, et al. Mesenchymal stem cell secretion of chemokines during differentiation into osteoblasts, and their potential role in mediating interactions with breast cancer cells. Int J Cancer. 2009;124(2):326-32.

24. Djouad F, Bouffi C, Ghannam S, Noel D, Jorgensen C. Mesenchymal stem cells: innovative therapeutic tools for rheumatic diseases. Nat Rev Rheumatol. 2009:5(7):392-9.

25. Hao ZC, Lu J, Wang SZ, Wu H, Zhang YT, Xu SG. Stem cell-derived exosomes: a promising strategy for fracture healing. Cell Prolif. 2017;e12359.

26. Lai RC, Arslan F, Lee MM, Sze NS, Choo A, Chen TS, et al. Exosome secreted by MSC reduces myocardial ischemia/reperfusion injury. Stem Cell Res. 2010;4:214-22.

27. Xin H, Li Y, Buller B, Katakowski M, Zhang Y, Wang X, et al. Exosome-mediated transfer of miR-133b from multipotent mesenchymal stromal cells to neural cells contributes to neurite out growth. Stem Cells. 2012;30:1556-64.

28. Li Y, Yang YY, Ren JL, Xu F, Chen FM, Li A. Exosomes secreted by stem cells from human exfoliated deciduous teeth contribute to functional recovery after traumatic brain injury by shifting microglia M1/M2 polarization in rats. Stem Cell Res Ther. 2017;8(1):198.

29. Bliss LA, Sams MR, Deep-Soboslay A, Ren-Patterson R, Jaffe AE, Chenoweth $J G$, et al. Use of postmortem human dura mater and scalp for deriving human fibroblast cultures. PLoS One. 2012;7(9):e45282.

30. Cao Y, Sun Z, Liao L, Meng Y, Han Q, Zhao RC. Human adipose tissuederived stem cells differentiate into endothelial cells in vitro and improve postnatal neovascularization in vivo. Biochem Biophys Res Commun. 2005; 332(2):370-9.

31. Botstein GR, Sherer GK, Leroy EC. Fibroblast selection in scleroderma: An alternative model of fibrosis. Arthritis Rheum. 1982;25:189-95.

32. Ohshima K, Inoue K, Fujiwara A, Hatakeyama K, Kanto K, Watanabe Y, et al. Let-7 microRNA family is selectively secreted into the extracellular environment via exosomes in a metastatic gastric cancer cell line. PLoS One. 2010;5(10):e13247.

33. Lin R, Wang $S$, Zhao RC. Exosomes from human adipose-derived mesenchymal stem cells promote migration through Wnt signaling pathway in a breast cancer cell model. Mol Cell Biochem. 2013;383(1-2):13-20.
34. Zhu L, Lang J, Liu C, et al. The epidemiological study of women with urinary incontinence and risk factors for stress urinary incontinence in China. Menopause. 2009;16:831-6.

35. Chen BH, Wen Y, Li H, Polan u. Collagen metabolism and turnover in women with stress urinary incontinence and pelvic prolapse. Int Urogynecol J Pelvic Floor Dysfunct. 2002;13(2):80-7.

36. Pittenger MF, Mackay AM, Beck SC, Jaiswal RK, Douglas R, Mosca JD, et al. Multilineage potential of adult human mesenchymal stem cells. Science. 1999:284:143-7

37. Teixeira FG, Carvalho MM, Sousa N, Salgado AJ. Mesenchymal stem cells secretome:a new paradigm for central nervous system regeneration? Cell Mol Life Sci. 2013;70:3871-82.

38. Timmers L, Lim SK, Arslan F, Armstrong JS, Hoefer IE, Doevendans PA, et al. Reduction of myocardial infarct size by human mesenchymal stem cell conditioned medium. Stem Cell Res. 2007;1:129-37.

39. Salgado AJ, Sousa JC, Costa BM, Pires AO, Mateus-Pinheiro A, Teixeira FG, et al. Mesenchymal stem cells secretome as a modulator of the neurogenic niche: basic insights and therapeutic opportunities. Front Cell Neurosci. 2015;9:249.

40. Konala VB, Mamidi MK, Bhonde R, Das AK, Pochampally R, Pal R. The current landscape of the mesenchymal stromal cell secretome: a new paradigm for cell-free regeneration. Cytotherapy. 2016:18:13-24.

41. Marote A, Teixeira FG, Mendes-Pinheiro B, Salgado AJ. MSCs-Derived Exosomes: Cell-Secreted Nanovesicles with Regenerative Potential. Front Pharmacol. 2016;7:231

42. Hu L, Wang J, Zhou $X$, Xiong Z, Zhao J, Yu R, et al. Exosomes derived from human adipose mensenchymal stem cells accelerates cutaneous wound healing via optimizing the characteristics of fibroblasts. Sci Rep. 2016;6:32993.

\section{Ready to submit your research? Choose BMC and benefit from:}

- fast, convenient online submission

- thorough peer review by experienced researchers in your field

- rapid publication on acceptance

- support for research data, including large and complex data types

- gold Open Access which fosters wider collaboration and increased citations

- maximum visibility for your research: over $100 \mathrm{M}$ website views per year

At BMC, research is always in progress.

Learn more biomedcentral.com/submissions 